\title{
Ferromagnetic Fixed Point of the Kondo Model in a Luttinger Liquid
}

\author{
Mun Dae Kim ${ }^{a, b}$, Chul Koo Kim ${ }^{a, b}, \mathrm{Kyun}_{\mathrm{Nahm}^{c}}$ and Chang-Mo Ryu ${ }^{d}$ \\ ${ }^{a}$ Institute of Physics and Applied Physics, Yonsei University, Seoul 120-749, Korea \\ ${ }^{b}$ Center for Strongly Correlated Materials Research, Seoul National University, Seoul 151-742, Korea \\ ${ }^{c}$ Department of Physics, Yonsei University, Wonju 220-710, Korea \\ ${ }^{d}$ Department of Physics, Pohang University of Science and Technology, Pohang 790-784, Korea
}

\begin{abstract}
The Kondo effect in a Luttinger liquid is studied using the renormalization group method. By renormalizing the boson fields, scaling equations to the second order for an arbitrary Luttinger interaction are obtained. For the ferromagnetic Kondo coupling, a spin bound state(triplet) can be realized without invoking a nearest neighbor spin interaction in agreement with the recent Bethe ansatz calculation. The scaling theory in the presence of the scalar potential shows that there is no interplay between the magnetic and non-magnetic interaction. Also a study on the crossover behavior of the Kondo temperature between the exponential and the power law type is presented.
\end{abstract}

\section{INTRODUCTION}

The Kondo problem in one-dimensional quantum system has attracted substantial interest in connection with the recent rapid development of the nanofabrication technology. In one-dimension, interacting electron system is described by the Tomonaga-Luttinger liquid theory [1] [- whose low- energy excitations are not quasiparticles but collective charge and spin density fluctuations. The magnetic impurity effect in this non-Fermi liquid was first studied by Lee and Toner using a scaling analysis on the kink gas action [6]. They obtained the scaling equation to the first order of coupling constant in the weak coupling regime. Furusaki and Nagaosa(FN) extended the study and obtained a set of scaling equation up to the second order using the poor man's scaling theory [7]. In their study, FN proposed an interesting conjecture that even ferromagnetic Kondo impurity as well as antiferromagnetic one would be completely screened. In the antiferromagnetic coupling case, the coupling constant flows to a strong coupling regime and, thus, the magnetic impurity and a conduction electron form a singlet. But, in the ferromagnetic case, situation is not clear whether it flows into a strong coupling regime or not. In order to clarify the situation, FN considered coupling of impurity spins not only with the same site electrons but also with the nearest neighbor conduction electrons. In this picture, the impurity and three conduction electrons form a singlet composite. However, a recent Bethe ansatz(BA) result by the Wang and Voit showed that spins form a triplet for the ferromagnetic coupling case [8]. These conflicting results call for a more detailed renormalization group(RG) analysis to clarify the physical situation.

In this paper, we carry out an RG analysis which goes beyond the poor man's scaling scheme in the presence of the Kondo interaction. We bosonize the Kondo interaction term using the Abelian bosonization. Carrying out a full RG calculation for the second order cumulant, we obtain a set of general scaling equations valid for an arbitrary strength of the Luttinger interaction. We show that the present RG calculation confirms the calculation drawn from the BA calculation using an open boundary condition.

Another recent BA study of the same problem by Wang and Eckern showed that there is a competition between the Kondo coupling and the impurity potential [9]. When the impurity potential is dominant, the system is shown to flow to a weak coupling fixed point. However, when the magnetic interaction is dominant, a spin complex is shown to be formed. In order to investigate this problem, we bosonize the potential scattering term and perform a scaling calculation. The result shows that the magnetic interaction and the potential scattering do not interplay in the scaling procedure and flow independently.

In the first order scaling, the Kondo temperature in the Luttinger can be calculated analytically to show a power-law behavior in contrast to the exponential one of the conventional Kondo model [6]. However, it is not possible to show the crossover from the Fermi to the Luttinger liquid in the first order. In this paper, we study the crossover behavior of the Kondo temperature from the exponential to the power-law type as a function of the Luttinger interaction, by solving the RG equations.

\section{BOSONIZATION OF THE KONDO HAMILTONIAN AND THE PARTITION FUNCTION}

Introducing the two phase fields,

$$
\phi_{\nu}=-\frac{i \pi}{L} \sum_{p \neq 0} \frac{e^{-\alpha|p| / 2-i p x}}{p}\left(\nu_{+}(p)+\nu_{-}(p)\right)-\left(N_{+\nu}+N_{-\nu}\right) \frac{\pi x}{L}
$$




$$
\theta_{\nu}=\frac{i \pi}{L} \sum_{p \neq 0} \frac{e^{-\alpha|p| / 2-i p x}}{p}\left(\nu_{+}(p)-\nu_{-}(p)\right)+\left(N_{+\nu}-N_{-\nu}\right) \frac{\pi x}{L},
$$

the one-dimensional model Hamiltonian with the forward electron-electron scattering is written by

$$
H=\frac{1}{2 \pi} \sum_{\nu} \int d x\left(v_{\nu} \eta_{\nu} \pi^{2} \pi_{\nu}^{2}(x)+\frac{v_{\nu}}{\eta_{\nu}}\left(\frac{\partial \phi_{\nu}(x)}{\partial x}\right)^{2}\right),
$$

where $\nu=\rho$ or $\sigma,+(-)$ means right(left) going mode, and the parameter $v_{\nu}$ and $\eta_{\nu}$ are given by

$$
v_{\nu}=\sqrt{\left(v_{F}+\frac{g_{4 \nu}}{\pi}\right)^{2}-\left(\frac{g_{2 \nu}}{\pi}\right)^{2}}, \quad \eta_{\nu}=\sqrt{\frac{v_{F}+\frac{g_{4 \nu}-g_{2 \nu}}{\pi}}{v_{F}+\frac{g_{4 \nu}+g_{2 \nu}}{\pi}}} .
$$

Here, $g_{2}$ and $g_{4}$ are the dimensionless coupling parameters [3]. The fields $\phi_{\nu}$ and $\pi_{\nu}$ satisfy the canonical boson commutation relation;

$$
\begin{aligned}
& {\left[\phi_{\nu}(x), \pi_{\nu^{\prime}}\left(x^{\prime}\right)\right]=i \delta_{\nu \nu^{\prime}} \delta\left(x-x^{\prime}\right),} \\
& {\left[\phi_{\nu}(x), \theta_{\nu^{\prime}}\left(x^{\prime}\right)\right]=i \frac{\pi}{2} \delta_{\nu \nu^{\prime}} \operatorname{sign}\left(x^{\prime}-x\right), \quad \theta_{\nu}(x)=\pi \int_{-\infty}^{x} \pi_{\nu}(y) d y .}
\end{aligned}
$$

Alternatively, this Hamiltonian can also be obtained directly from the fermion representation of the one dimensional interacting electron system using

$$
\psi_{r s}(x)=\lim _{\alpha \rightarrow 0} \frac{e^{i r\left(k_{F}-\frac{\pi}{L}\right) x}}{\sqrt{2 \pi \alpha}} \eta_{r s} e^{-\frac{i}{\sqrt{2}}\left(r \phi_{\rho}(x)-\theta_{\rho}(x)+s\left(r \phi_{\sigma}(x)-\theta_{\sigma}(x)\right)\right)},
$$

where $\eta_{r s}$ is the Majorana fermion operator which satisfies the following relations [5];

$$
\begin{aligned}
{\left[\eta_{r s}, \eta_{r^{\prime} s^{\prime}}\right] } & =2 \delta_{r r^{\prime}} \delta_{s s^{\prime}} \\
\eta_{+\downarrow} \eta_{+\uparrow}=\eta_{-\downarrow} \eta_{-\uparrow}, \quad \eta_{+\downarrow} \eta_{-\uparrow} & =-\eta_{-\downarrow} \eta_{+\uparrow}, \quad \eta_{+\uparrow} \eta_{-\uparrow}=-\eta_{+\downarrow} \eta_{-\downarrow} \quad \ldots
\end{aligned}
$$

If a single magnetic impurity is introduced in one-dimensional interacting electron system, the Kondo interaction term is given by

$$
\begin{aligned}
H_{K} & =J \vec{S} \cdot \vec{s}(0) \\
& =J_{z} S_{z} s_{z}(0)+\frac{1}{2} J_{\perp}\left(S_{+} s_{-}(0)+S_{-} s_{+}(0)\right),
\end{aligned}
$$

where $\vec{s}=\frac{1}{2} \sum_{r r^{\prime} \sigma \sigma^{\prime}} \psi_{r \sigma}^{\dagger} \vec{\sigma}_{\sigma \sigma^{\prime}} \psi_{r^{\prime} \sigma^{\prime}}$ and $s_{ \pm}=s_{x} \pm i s_{y}$. Using the relations of the Majorana fermions, Eq.(6), and the bosonization formula of the fermion operators, we obtain the bosonized Kondo Hamiltonian [10],

$$
\begin{aligned}
H_{K} & =J \vec{S} \cdot \vec{s} \\
& =\frac{S_{z}}{2 \pi \alpha}\left(J_{z F} \alpha \partial_{x} \sqrt{2} \phi_{\sigma}(0)+2 i J_{z B} \eta_{+\uparrow} \eta_{-\uparrow} \sin \left(\sqrt{2} \phi_{\rho}(0)\right) \cos \left(\sqrt{2} \phi_{\sigma}(0)\right)\right) \\
& +\frac{S_{+}}{2 \pi \alpha} e^{\sqrt{2} i \theta_{\sigma}(0)}\left(J_{\perp F} \eta_{+\downarrow} \eta_{+\uparrow} \cos \left(\sqrt{2} \phi_{\sigma}(0)\right)+i J_{\perp B} \eta_{+\downarrow} \eta_{-\uparrow} \sin \left(\sqrt{2} \phi_{\rho}(0)\right)\right) \\
& +\frac{S_{-}}{2 \pi \alpha} e^{-\sqrt{2} i \theta_{\sigma}(0)}\left(J_{\perp F} \eta_{+\uparrow} \eta_{+\downarrow} \cos \left(\sqrt{2} \phi_{\sigma}(0)\right)-i J_{\perp B} \eta_{-\uparrow} \eta_{+\downarrow} \sin \left(\sqrt{2} \phi_{\rho}(0)\right)\right) .
\end{aligned}
$$

The partition function of the system at temperature $T=1 / \beta$ is

$$
\begin{aligned}
Z=\int & D \phi_{\rho} D \phi_{\sigma} D \theta_{\rho} D \theta_{\sigma} e^{-S} \\
S & =\int d x \int d \tau\left(L_{0}+L_{K}\right), \\
L_{0} & =\sum_{\nu=\rho, \sigma}\left(i \partial_{\tau} \phi_{\nu}(x, \tau) \pi_{\nu}(x, \tau)+\frac{1}{2 \pi}\left(v_{\nu} \eta_{\nu} \pi^{2} \pi_{\nu}^{2}(x, \tau)+\frac{v_{\nu}}{\eta_{\nu}}\left(\frac{\partial \phi_{\nu}(x, \tau)}{\partial x}\right)^{2}\right)\right), \\
L_{K} & =H_{K}\left(\phi_{\nu}(0, \tau), \theta_{\nu}(0, \tau)\right),
\end{aligned}
$$

where the integration is over the bosonic fields $\phi_{\nu}(x, \tau)$ and $\theta_{\nu}(x, \tau)$ with imaginary time $\tau$ running from 0 to $\beta$. 


\section{RENORMALIZATION ANALYSIS}

First, we divide the phase fields $\phi_{\nu}$ into slow and fast mode:

$$
\begin{aligned}
\phi_{\nu}(\tau)=\phi_{\nu s}(\tau) & +\phi_{\nu f}(\tau) \\
\phi_{\nu s}(\tau) & =\frac{1}{\beta} \sum_{\left|\omega_{n}\right|<\mu} \tilde{\phi}_{\nu}(\omega) e^{-i \omega \tau} \\
\phi_{\nu f}(\tau) & =\frac{1}{\beta} \sum_{\mu<\left|\omega_{n}\right|<\lambda} \tilde{\phi}_{\nu}(\omega) e^{-i \omega \tau} .
\end{aligned}
$$

The average over the fast mode of the partition function is carried out, using the cumulant expansion,

$$
\begin{aligned}
Z & =Z_{0}<e^{-S_{K}}>_{0} \\
& =Z_{0} \int D \phi_{\nu s} D \theta_{\nu s} e^{-S_{0}\left(\phi_{\nu s}, \theta_{\nu s}\right)} e^{-<S_{K}>_{0}^{f}+\frac{1}{2}\left(<S_{K}^{2}>_{0}^{f}-<S_{K}>_{0}^{f^{2}}\right)+\cdots}
\end{aligned}
$$

where $Z_{0}=\int D \phi_{\nu} D \theta_{\nu} e^{-S_{0}}$, f indicates an average over the fast mode which will be omitted hereafter, and $<\cdots>_{0}$ represents an average over $Z_{0}$.

First, we consider the first order forward longitudinal scattering term,

$$
<\int d \tau J_{z F} \alpha \partial_{x} \sqrt{2} \phi_{\sigma}(\tau)>=\int d \tau J_{z F} \alpha\left(\partial_{x} \sqrt{2} \phi_{\sigma s}(\tau)+<\partial_{x} \sqrt{2} \phi_{\sigma f}(\tau)>\right) .
$$

The second term in the right side vanishes because it is an average of an odd function. We, thus, conclude that $\delta J_{z F}=0$ in the first order. The backward longitudinal scattering part is scaled as follows,

$$
\begin{array}{r}
\int d \tau J_{z B} \eta_{+\uparrow} \eta_{-\uparrow}<\sin \left(\sqrt{2} \phi_{\rho}(\tau)><\cos \left(\sqrt{2} \phi_{\sigma}(\tau)\right)>\right. \\
=\left(\frac{\mu}{\lambda}\right)^{\frac{\eta_{\rho}}{2}+\frac{\eta_{\sigma}}{2}} \int d \tau J_{z B} \eta_{+\uparrow} \eta_{-\uparrow} \sin \left(\sqrt{2} \phi_{\rho s}(\tau) \cos \left(\sqrt{2} \phi_{\sigma s}(\tau)\right) .\right.
\end{array}
$$

In the above, we utilized the fact that the charge and spin degrees are separated. The rescaling procedure,

$$
J_{z B}(\mu)=\left(\frac{\mu}{\lambda}\right)^{\frac{\eta_{\rho}+\eta_{\sigma}}{2}-1} J_{z B}(\lambda)
$$

gives

$$
\frac{\delta J_{z B}}{J_{z B}}=\left(\frac{\eta_{\rho}+\eta_{\sigma}}{2}-1\right) \frac{\delta \lambda}{\lambda}
$$

where $\mu=\lambda+\delta \lambda$ and $\delta l=-\frac{\delta \lambda}{\lambda}=-\delta \ln \lambda$. Thus, we have in the first order

$$
\begin{aligned}
& \frac{d J_{z F}}{d l}=0 \\
& \frac{d J_{z B}}{d l}=\left(1-\frac{\eta_{\rho}+\eta_{\sigma}}{2}\right) J_{z B} .
\end{aligned}
$$

The scaling equations for the other scattering terms can be similarly obtained;

$$
\begin{aligned}
& \frac{d J_{\perp F}}{d l}=\left[1-\left(\frac{1}{2 \eta_{\sigma}}+\frac{\eta_{\sigma}}{2}\right)\right] J_{\perp F}, \\
& \frac{d J_{\perp B}}{d l}=\left[1-\left(\frac{1}{2 \eta_{\sigma}}+\frac{\eta_{\rho}}{2}\right)\right] J_{\perp B} .
\end{aligned}
$$

These equations are in agreement with those of Lee and Toner [6].

The second order cumulant is given by $-\frac{1}{2}\left(<S_{K}^{2}>-<S_{K}>^{2}\right)$, where $<S_{K}>^{2}$ term is to eliminate unconnected diagrams. We consider one of the $J_{z B} J_{\perp F}$ terms which is given by 


$$
\begin{aligned}
\int d \tau \int d \tau^{\prime} & \frac{S_{z} S_{+}}{(2 \pi \alpha)^{2}} J_{z B} 2 i \eta_{+\uparrow} \eta_{-\uparrow} J_{\perp F} \eta_{+\downarrow} \eta_{+\uparrow} \\
& \left(<\sin \left(\sqrt{2} \phi_{\rho}(\tau)\right) \cos \left(\sqrt{2} \phi_{\sigma}(\tau)\right) e^{\sqrt{2} i \theta_{\sigma}\left(\tau^{\prime}\right)} \cos \left(\sqrt{2} \phi_{\sigma}\left(\tau^{\prime}\right)\right)>\right. \\
& \left.-<\sin \left(\sqrt{2} \phi_{\rho}(\tau)\right) \cos \left(\sqrt{2} \phi_{\sigma}(\tau)\right)><e^{\sqrt{2} i \theta_{\sigma}\left(\tau^{\prime}\right)} \cos \left(\sqrt{2} \phi_{\sigma}\left(\tau^{\prime}\right)\right)>\right) .
\end{aligned}
$$

In order to evaluate this expression, we need the two point correlation function 11

$$
\begin{aligned}
G(x, \tau) & =<\phi(x, \tau) \phi(0,0)> \\
& =\int \frac{d q}{2 \pi} \int \frac{d \omega}{2 \pi} e^{-i q x} e^{i \omega \tau} \frac{\pi}{\frac{1}{v \eta} \omega^{2}+\frac{v}{\eta} q^{2}} \\
G(\tau) \equiv & G(0, \tau) \\
& = \begin{cases}\frac{\eta}{2} K_{0}(\mu \tau) & \text { for } \lambda \tau>>1 \\
\frac{\eta}{2} \ln \frac{\lambda}{\mu} & \text { for } \lambda \tau<<1,\end{cases}
\end{aligned}
$$

where $K_{0}$ is the modified Bessel function of the second kind. $G(\tau)$ decays exponentially with the renormalized lattice spacing $\frac{1}{\mu}$, and decreases logarithmically for small $\lambda \tau$. Thus, we regard $G(\tau)$ short ranged and, thus, expand the cosine terms around zero. It is known that the higher harmonics $\cos (2 \sqrt{2} \phi(0))$ is irrelevant and $\left(\left.(\partial \phi(\tau) / \partial \tau)\right|_{\tau=0}\right)^{2}$ terms which is the most relevant term in the expansion can also be shown irrelevant by the power counting [11]. Short time cut off, $\tau_{0} \sim \alpha / v_{F}$, merely introduces an overall constant which does not affect the flow of the parameter. Therefore Eq.(18) is reduced to

$$
\int d \tau \frac{\alpha}{v_{F}} \frac{1}{(2 \pi \alpha)^{2}} \frac{1}{2} S_{+} J_{z B} J_{\perp F}\left(-i \eta_{+\downarrow} \eta_{-\uparrow}\right)\left(\frac{\mu}{\lambda}\right)^{\frac{\eta_{\rho}}{2}+\frac{1}{2 \eta_{\sigma}}+\eta_{\sigma}}\left(\left(\frac{\mu}{\lambda}\right)^{-\eta_{\sigma}}-1\right) e^{\sqrt{2} i \theta_{\sigma}(\tau)} \sin \left(\sqrt{2} \phi_{\rho}(\tau)\right) .
$$

Collecting other terms of the second order cumulant and rescaling as before, we have for the transverse backward part,

$$
\int d \tau \frac{S_{+}}{2 \pi \alpha} i \eta_{+\downarrow} \eta_{-\uparrow} \eta_{\sigma} J_{z B} J_{\perp F} \frac{d \lambda}{\lambda} e^{\sqrt{2} i \theta_{\sigma s}(\tau)} \sin \left(\sqrt{2} \phi_{\rho s}(\tau)\right),
$$

which renormalize the coupling constant $J_{\perp B}$ of the Kondo term. Similarly, we have for the transverse forward scattering part which renormalizes the coupling constant $J_{\perp F}$,

$$
\int d \tau \frac{S_{+}}{2 \pi \alpha} \eta_{+\downarrow} \eta_{+\uparrow} \eta_{\rho} J_{z B} J_{\perp B} \frac{d \lambda}{\lambda} e^{\sqrt{2} i \theta_{\sigma}(\tau)} \cos \left(\sqrt{2} \phi_{\sigma}(\tau)\right) .
$$

The same scaling process on the $S_{-}$terms gives the same renormalization for both the $J_{\perp B}$ and $J_{\perp F}$.

However, the scaling process involving the descendant field terms, $J_{z F} \alpha \partial_{x} \sqrt{2} \phi_{\sigma}(\tau)$, is somewhat different. One example of such a term is the second order cumulant for the transverse forward scattering, which is given by

$$
\begin{array}{r}
\frac{S_{z} S_{+}}{(2 \pi \alpha)^{2}} J_{z F} J_{\perp F} \eta_{+\downarrow} \eta_{+\uparrow}\left(<\alpha \partial_{x} \sqrt{2} \phi_{\sigma}(\tau) e^{i \sqrt{2} \theta_{\sigma}\left(\tau^{\prime}\right)} \cos \left(\sqrt{2} \phi_{\sigma}\left(\tau^{\prime}\right)>\right.\right. \\
-<\alpha \partial_{x} \sqrt{2} \phi_{\sigma}(\tau)><e^{i \sqrt{2} \theta_{\sigma}\left(\tau^{\prime}\right)} \cos \left(\sqrt{2} \phi_{\sigma}\left(\tau^{\prime}\right)\right)> \\
=\frac{S_{z} S_{+}}{(2 \pi \alpha)^{2}} J_{z F} J_{\perp F} \eta_{+\downarrow} \eta_{+\uparrow}<\alpha \partial_{x} \sqrt{2} \phi_{\sigma f}(\tau) e^{i \sqrt{2} \theta_{\sigma}\left(\tau^{\prime}\right)} \cos \left(\sqrt{2} \phi_{\sigma}\left(\tau^{\prime}\right)\right)>.
\end{array}
$$

Here, we note that

$$
\begin{aligned}
& <\alpha \partial_{x} \sqrt{2} \phi_{\sigma f}(\tau) e^{i \sqrt{2} \theta_{\sigma}\left(\tau^{\prime}\right)} e^{i \sqrt{2} \phi_{\sigma}\left(\tau^{\prime}\right)}> \\
= & \lim _{\epsilon \rightarrow 0} \frac{1}{i \epsilon} \frac{\partial}{\partial x}<\alpha e^{i \epsilon \sqrt{2} \phi_{\sigma f}(x, \tau)} e^{i \sqrt{2} \theta_{\sigma}\left(0, \tau^{\prime}\right)} e^{i \sqrt{2} \phi_{\sigma}\left(0, \tau^{\prime}\right)}>\left.\right|_{x=0} .
\end{aligned}
$$

Using $e^{A+B}=e^{A} e^{B} e^{-\frac{1}{2}[A, B]}$, the above expression takes a form,

$$
\left.\frac{\alpha}{i}\left(2 \partial_{x} G_{\phi_{\sigma f}}(x, \tau)-2 \partial_{x}<\phi_{\sigma f}(x, \tau) \theta_{\sigma f}(0,0)>\right)\right|_{x=0} e^{-G_{\theta_{\sigma f}}(0,0)-G_{\theta_{\phi f}}(0,0)} .
$$


In order to calculate $\partial_{x}<\phi_{\sigma f}(x, \tau) \theta_{\sigma f}(0,0)>$, we use the relations between density field, $\phi_{\sigma}$, and current field, $\theta_{\sigma}$ [3, 14];

$$
\begin{aligned}
& -\frac{i}{\eta_{\sigma}} \frac{\partial \phi_{\sigma}}{\partial\left(v_{\sigma} \tau\right)}=\frac{\partial \theta_{\sigma}}{\partial x} \\
& -i \eta_{\sigma} \frac{\partial \theta_{\sigma}}{\partial\left(v_{\sigma} \tau\right)}=\frac{\partial \phi_{\sigma}}{\partial x} .
\end{aligned}
$$

Then, we have

$$
\begin{aligned}
\alpha \partial_{x}<\phi_{\sigma f}(x, \tau) \theta_{\sigma f}(0,0)> & =\alpha \eta_{\sigma} \frac{\partial}{\partial\left(v_{\sigma} \tau\right)}<\theta_{\sigma f}(x, \tau) \theta_{\sigma f}(0,0)> \\
& =\frac{1}{2} \frac{d \lambda}{\lambda}
\end{aligned}
$$

using Eq.(19) with $\frac{1}{\eta} \rightarrow \eta$ and the short-ranged nature of the correlation function $G(\tau)$. Substituting this result into Eq.(23), we obtain

$$
\begin{aligned}
& \frac{1}{2 \pi \alpha} S_{+} \eta_{+\downarrow} \eta_{+\uparrow}\left(\frac{1}{2 \pi v_{F}} J_{z F} J_{\perp F} \frac{d \lambda}{\lambda}\right) e^{i \sqrt{2} \theta_{\sigma}(\tau)} \cos \left(\sqrt{2} \phi_{\sigma}(\tau)\right) \\
+ & \frac{1}{2 \pi \alpha} S_{+} \eta_{+\downarrow} \eta_{-\uparrow}\left(\frac{1}{2 \pi v_{F}} J_{z F} J_{\perp B} \frac{d \lambda}{\lambda}\right) e^{i \sqrt{2} \theta_{\sigma}(\tau)} \sin \left(\sqrt{2} \phi_{\rho}(\tau)\right),
\end{aligned}
$$

for the renormalization of $J_{\perp F}$ and $J_{\perp B}$.

The longitudinal scattering parameter is scaled by consecutive transverse scattering processes. The forward scattering part which contains $J_{\perp F}^{2}$ is

$$
\begin{array}{r}
\frac{S_{+} S_{-}}{(2 \pi \alpha)^{2}} J_{\perp F}^{2} \eta_{+\downarrow} \eta_{+\uparrow} \eta_{+\uparrow} \eta_{+\downarrow}\left[<e^{\sqrt{2} i \theta_{\sigma}(\tau)} \cos \left(\sqrt{2} \phi_{\sigma}(\tau)\right) e^{-\sqrt{2} i \theta_{\sigma}\left(\tau^{\prime}\right)} \cos \left(\sqrt{2} \phi_{\sigma}\left(\tau^{\prime}\right)\right)>\right. \\
\left.-<e^{\sqrt{2} i \theta_{\sigma}(\tau)} \cos \left(\sqrt{2} \phi_{\sigma}(\tau)\right)><e^{-\sqrt{2} i \theta_{\sigma}\left(\tau^{\prime}\right)} \cos \left(\sqrt{2} \phi_{\sigma}\left(\tau^{\prime}\right)\right)>\right] .
\end{array}
$$

The typical relevant term is given by

$$
e^{\sqrt{2} i \theta_{\sigma}(\tau)} e^{-\sqrt{2} i \theta_{\sigma}\left(\tau^{\prime}\right)} e^{-\sqrt{2} i \phi_{\sigma}(\tau)} e^{\sqrt{2} i \phi_{\sigma}\left(\tau^{\prime}\right)} .
$$

Other terms containing higher harmonics, $e^{2 \sqrt{2} i \theta_{\sigma}(\tau)}$ or $e^{2 \sqrt{2} i \phi_{\sigma}(\tau)}$ are irrelevant 11, 15. Separating the fields into the fast and slow mode, we average on the fast mode to obtain a short range correlation and expand the slow mode to obtain

$$
1+\left.i \sqrt{2} \partial_{\tau} \theta_{\sigma s}(\tau)\right|_{\tau=0} \tau+\left.i \sqrt{2} \partial_{\tau} \phi_{\sigma s}(\tau)\right|_{\tau=0} \tau+\cdots
$$

We note that $\partial_{\tau} \theta_{\sigma s}(\tau)$ gives the longitudinal scattering contribution, $\partial_{x} \phi_{\sigma s}$, through Eq.(26), while $\partial_{\tau} \phi_{\sigma s}$ term is canceled by other terms. Including the contributions from the $S_{-} S_{+}$term, we obtain

$$
\begin{gathered}
\int d \tau \\
{\left[\frac{1}{2 \pi \alpha} \frac{S_{z}}{2 \pi v_{F}} \frac{1}{\eta_{\sigma}}\left(\frac{1}{2 \eta_{\sigma}}+\frac{\eta_{\sigma}}{2}\right) J_{\perp F}^{2} \frac{d \lambda}{\lambda} \alpha \partial_{x} \sqrt{2} \phi_{\sigma s}(\tau)\right.} \\
\left.+\frac{1}{2 \pi \alpha} \frac{S_{z}}{2 \pi v_{F}} \frac{1}{\eta_{\sigma}}\left(\frac{1}{2 \eta_{\sigma}}+\frac{\eta_{\rho}}{2}\right) J_{\perp B}^{2} \frac{d \lambda}{\lambda} \alpha \partial \sqrt{2} \phi_{\sigma s}(\tau)\right]
\end{gathered}
$$

for the $J_{\perp B}^{2}$ contribution to the $J_{z F}$ renormalization. Consecutive transverse scattering also renormalizes the longitudinal backward term, $J_{z B}$, similarly;

$$
\int d \tau \frac{S_{z}}{2 \pi \alpha} 2 i \eta_{+\uparrow} \eta_{-\uparrow} \frac{2}{2 \pi v_{F}} \eta_{\sigma} J_{\perp F} J_{\perp B} \frac{d \lambda}{\lambda} \sin \left(\sqrt{2} \phi_{\rho}(\tau)\right) \cos \left(\sqrt{2} \phi_{\sigma}(\tau)\right) .
$$

The last term to be considered in the second order cumulant expansion is the $S_{z}^{2} J_{z F}^{2}$ term. Scaling of this term, however, does not contribute to the magnetic interaction and, also, does not produce any relevant term for nonmagnetic interaction. Collecting the results together, we now have the scaling equations to the second order; 


$$
\begin{aligned}
& \frac{d J_{z F}}{d l}=\quad \frac{1}{2 \pi v_{F}}\left(\frac{1}{2 \eta_{\sigma}}\left(\frac{1}{\eta_{\sigma}}+\eta_{\sigma}\right) J_{\perp F}^{2}+\frac{1}{2 \eta_{\sigma}}\left(\frac{1}{\eta_{\sigma}}+\eta_{\rho}\right) J_{\perp B}^{2}\right), \\
& \frac{d J_{z B}}{d l}=\left(1-\frac{\eta_{\sigma}-\eta_{\rho}}{2}\right) J_{z B}+\frac{1}{2 \pi v_{F}} 2 \eta_{\sigma} J_{\perp F} J_{\perp B}, \\
& \frac{d J_{\perp F}}{d l}=\left(1-\frac{\eta_{\sigma}+\frac{1}{\eta_{\sigma}}}{2}\right) J_{\perp F}+\frac{1}{2 \pi v_{F}}\left(J_{z F} J_{\perp F}+\eta_{\rho} J_{z B} J_{\perp B}\right), \\
& \frac{d J_{\perp B}}{d l}=\left(1-\frac{\eta_{\sigma}-\eta_{\rho}}{2}\right) J_{\perp B}+\frac{1}{2 \pi v_{F}}\left(J_{z F} J_{\perp B}+\eta_{\sigma} J_{z B} J_{\perp F}\right) .
\end{aligned}
$$

These equations can be simplified assuming the $\mathrm{SU}(2)$ symmetry for the conduction electrons $\left(\eta_{\sigma}=1\right)$;

$$
\begin{array}{rr}
\frac{d\left(\rho_{0} J_{z F}\right)}{d l}= & \left(\rho_{0} J_{\perp F}\right)^{2}+\left(\frac{1+\eta_{\rho}}{2}\right)\left(\rho_{0} J_{\perp B}\right)^{2} \\
\frac{d\left(\rho_{0} J_{z B}\right)}{d l}=\left(\frac{1-\eta_{\rho}}{2}\right)\left(\rho_{0} J_{z B}\right)+ & 2\left(\rho_{0} J_{\perp F}\right)\left(\rho_{0} J_{\perp B}\right) \\
\frac{d\left(\rho_{0} J_{\perp F}\right)}{d l}= & \left(\rho_{0} J_{z F}\right)\left(\rho_{0} J_{\perp F}\right)+\eta_{\rho}\left(\rho_{0} J_{z B}\right)\left(\rho_{0} J_{\perp B}\right) \\
\frac{d\left(\rho_{0} J_{\perp B}\right)}{d l}=\left(\frac{1-\eta_{\rho}}{2}\right)\left(\rho_{0} J_{\perp B}\right)+ & \left(\rho_{0} J_{z F}\right)\left(\rho_{0} J_{\perp B}\right)+\left(\rho_{0} J_{z B}\right)\left(\rho_{0} J_{\perp F}\right),
\end{array}
$$

where $\rho_{0}\left(=1 / 2 \pi v_{F}\right)$ is proportional to the density of state. As we see later, for ferromagnetic $\mathrm{J}$, the triplet ground state can be formed due to the interacton parameter $\eta_{\rho}$ in the second order term. In 1D correlated electron system, the electron-electron interaction induces a short range magnetic ordering which, in turn, introduces a molecular field on the magnetic impurity [16]. Therefore, the impurity and a electron form a triplet aligned to the molecular field, thus, resulting the broken local $\mathrm{SU}(2)$ symmetry. These equations are naturally reduced to the scaling equations of the conventional Kondo model when there is no Luttinger interaction i.e. $\eta_{\sigma}=1$ and $\eta_{\rho}=1$.

So far, no assumption has been made on the strength of $\eta_{\rho}\left(=\left[\left(1-g / \pi v_{F}\right) /\left(1+g / \pi v_{F}\right)\right]^{\frac{1}{2}}\right)$. Here, $\mathrm{g}$ is the strength of scattering between the right-going and the left-going mode and the scattering within the same mode is neglected. In the small $g$ regime, the above scaling equations are reduced as follows,

$$
\begin{aligned}
& \frac{d\left(\rho_{0} J_{z F}\right)}{d l}=\quad\left(\rho_{0} J_{\perp F}\right)^{2}+\left(\rho_{0} J_{\perp B}\right)^{2}-\frac{g}{2 \pi v_{F}}\left(\rho_{0} J_{\perp B}\right)^{2} \\
& \frac{d\left(\rho_{0} J_{z B}\right)}{d l}=\frac{g}{2 \pi v_{F}}\left(\rho_{0} J_{z B}\right)+2\left(\rho_{0} J_{\perp F}\right)\left(\rho_{0} J_{\perp B}\right) \\
& \frac{d\left(\rho_{0} J_{\perp F}\right)}{d l}=\quad\left(\rho_{0} J_{z F}\right)\left(\rho_{0} J_{\perp F}\right)+\left(\rho_{0} J_{z B}\right)\left(\rho_{0} J_{\perp B}\right)-\frac{2 g}{2 \pi v_{F}}\left(\rho_{0} J_{z B}\right)\left(\rho_{0} J_{\perp B}\right) \\
& \frac{d\left(\rho_{0} J_{\perp B}\right)}{d l}=\frac{g}{2 \pi v_{F}}\left(\rho_{0} J_{\perp B}\right)+\left(\rho_{0} J_{z F}\right)\left(\rho_{0} J_{\perp B}\right)+\left(\rho_{0} J_{z B}\right)\left(\rho_{0} J_{\perp F}\right) .
\end{aligned}
$$

We note that the above result is in agreement with the FN's poor man's scaling result except for the symmetry breaking terms involving the g parameter. In fact, the extra terms in our RG equations correspond to the next higher order terms which were neglected in the FN approach.

\section{THE GROUND STATE}

The scaling equations yield two strong coupling fixed points; $\left(J_{F}, J_{B}\right)=(\infty, \infty)$ and $(\infty,-\infty)$. The first fixed point governs the antiferromagnetic regime, which gives the singlet as the ground state. The second one corresponds to the ferromagnetic coupling. However it is not clear whether this fixed point corresponds to a singlet or to a triplet state. We have calculated flows of the coupling constant for several values of the Luttinger interaction parameter, $\eta$, for the ferromagnetic fixed point. For finite $\eta$ other than unity, $J_{B} / J_{F}<-1$ and $J_{B}+J_{F}$ flows to $-\infty$, whereas $J_{B}+J_{F}$ is equal to zero when $\eta=1$ (Fig. 1). When the coupling constant $J$ grows and becomes large, the Kondo coupling term becomes dominant in Eq.(35). Therefore, the Luttinger interaction becomes irrelevant [7] and we can treat the impurity spin as a classical spin with the magnitude $S=1 / 2$.

The asymtotic Hamiltonian which represents this situation can be written as 


$$
H=\sum_{r k \sigma} \epsilon_{k} c_{r k \sigma}^{\dagger} c_{r k \sigma}+\frac{1}{4} \sum_{r k, r^{\prime} k^{\prime}, \sigma} J_{r r^{\prime} \sigma} c_{r k \sigma}^{\dagger} c_{r^{\prime} k^{\prime} \sigma^{\prime}}
$$

where $J_{11 \sigma}=J_{22 \sigma}=\sigma J_{F}$ and $J_{12 \sigma}=J_{21 \sigma}=\sigma J_{B}$. The Luttinger interaction effect is included in the coupling constant through the renormalization process. The corresponding Green's functions are given as follows ;

$$
\begin{aligned}
& G_{11}(\epsilon)=G_{22}(\epsilon)=G_{0}(\epsilon)+G_{0}(\epsilon) \frac{\frac{1}{4} \sigma J_{F}}{1-\frac{1}{4} \sigma\left(J_{F}+J_{B}\right) G_{0}(\epsilon)} G_{0}(\epsilon), \\
& G_{12}(\epsilon)=G_{21}(\epsilon)=\quad G_{0}(\epsilon) \frac{\frac{1}{4} \sigma J_{B}}{1-\frac{1}{4} \sigma\left(J_{F}+J_{B}\right) G_{0}(\epsilon)} G_{0}(\epsilon) .
\end{aligned}
$$

For $\eta=1\left(J_{F}+J_{B}=0\right)$, there exist no bound state since the Green's functions do not have poles. This case has the same properties as the three-dimensional ferromagnetic Kondo coupling case. However, if the Luttinger interaction is turned on $(\eta<1), J_{F}+J_{B}$ flows to $-\infty$. The Green's functions have a pole for $\sigma=+1$ suggesting that a conduction electron becomes bounded [17] to form a triplet state in the ground state for the ferromagnetic Kondo exchange.

\section{THE SCALAR POTENTIAL SCATTERING EFFECT}

In a more realistic model, a magnetic impurity generates scattering due to an elastic potential, $\omega$ [10]. Recently,

Wang and his coworkers have carried out Bethe ansatz calculations on the one-dimensional Kondo problem [8.9]. They showed that for an attractive potential scattering, the bound state always appears, whereas for a repulsive potential scattering, there exists a bound state for $|J|>4 \omega$, but no bound state for $|J|<4 \omega$.

The scaling theory for the scalar potential in the first order is similar to the magnetic interaction. The bosonized term is

$$
\frac{1}{2 \pi \alpha}\left(2 \omega_{F} \alpha \partial_{x} \sqrt{2} \phi_{\rho}(0)+4 i \omega_{B} \eta_{+\uparrow} \eta_{-\uparrow} \cos \left(\sqrt{2} \phi_{\rho}(0)\right) \sin \left(\sqrt{2} \phi_{\sigma}(0)\right)\right)
$$

and we have the scaling equations

$$
\frac{d \omega_{F}}{d l}=0, \quad \frac{d \omega_{B}}{d l}=\frac{1-\eta_{\rho}}{2} \omega_{B}
$$

showing that the backscattering contribution is relevant. In the second order, the candidates for renormalizing the forward potential scattering are contributions from the terms like $\omega_{F}^{2}$ or $\omega_{B}^{2}$. But, we find that $\omega_{F}^{2}$ yields only a constant by a simple calculation,

$$
\frac{1}{(2 \pi \alpha)^{2}} 4 \omega_{F}^{2} \alpha^{2} \int d \tau \int d \tau^{\prime}<\partial_{x} \sqrt{2} \phi_{\rho_{f}}(\tau) \partial_{x} \sqrt{2} \phi_{\rho_{f}}\left(\tau^{\prime}\right)>
$$

which clearly has no slow mode. Similarly, the $\omega_{B}^{2}$ term generates terms such as $\cos \left(2 \sqrt{2} \phi_{\sigma}(\tau)\right)$, and $\cos \left(2 \sqrt{2} \phi_{\rho}(\tau)\right)$, which are irrelevant as discussed above. Also, we can show that $J_{z F}^{2}, J_{z B}^{2}, J_{z F} J_{z B}, w_{F} w_{B}$ and $J_{z \perp, F B} \omega_{F B}$ are also irrelevant through the same procedure as in $\omega_{F}^{2}$ and $\omega_{B}^{2}$. Thus, the potential scattering is not scaled at the second order cumulant and there is no interplay between the magnetic and the non-magnetic interaction. Actually, this situation is not unexpected, since we know that the nearest neighbor coulomb interaction in the Hubbard model does not affect the magnetic properties [18].

In the case of an impurity spin, $\mathrm{S}=1 / 2$, the energy of a single electron coupled with the impurity is such that the ferromagnetic coupling energy is $\frac{1}{4} J$ and the scalar potential is $\omega$. It means that if $4 \omega>|J|$ initially, the potential energy grows infinitely along the scaling process so that no bound state can be formed. If $4 \omega<|J|$ initially, a spin triplet state is formed. Under the open boundary condition, this corresponds to a bound state of a spin $\frac{3}{2}$ complex in agreement with the Bethe ansatz results [8]. Here, we note that, in the present treatment, it is not necessary to invoke the nearest neighbor spin interaction as done by FN. We believe that the short-ranged nature of the spin interaction makes the FN scenario unlikely, although it cannot be ruled out completely. 


\section{THE KONDO TEMPERATURE}

In the conventional three-dimensional Kondo model, the Kondo temperature is given by $T_{K}=D e^{-\frac{1}{2 J}}$, which originates from the scaling equation $d J / d \ln D=-2 J^{2}$, where $\mathrm{D}$ is the bandwidth. In their previous study, Lee and Toner showed that the scaling relation, $d J_{B} / d \ln D=-\frac{1-\eta}{2} J_{B}$, gives a power law Kondo temperature, $T_{K}=D J_{B}^{\frac{2}{1-\eta}}[6]$. However, the crossover behavior from the Fermi liquid to the Luttinger one has not been studied. In order to address this question, we consider the scaling to second order for the forward and the backward scattering simultaneously.

From the RG flow, we get the coupling constants as functions of $D$, i.e. $\tilde{J}_{F}(\tilde{D})$ and $\tilde{J}_{B}(\tilde{D})$, where $\tilde{J}_{F}$ and $\tilde{J}_{B}$ are the scaled couplings and $\tilde{D}$ is the scaled bandwidth cutoff. The Kondo temperature is an invariant energy scale in the scaling procedure and, thus, can be expressed as $T_{K}=\tilde{D} f(\tilde{J})$, where $f(\tilde{J})$ becomes exponential or power law type depending on the limiting case. For some initial value of $J, T_{K}$ remains constant through the scaling procedure. In such a case, $f(\tilde{J})$ becomes proportional to $\frac{1}{\tilde{D}(\tilde{J})}$, thus yielding the Kondo temperature.

$T_{K}$ for the backward scattering part is given in Fig. 2. It can be clearly seen that $T_{K}$ is exponential for a weak Luttinger interaction and becomes power law type as the Luttinger interaction increases. From inset, we observe that the linear slope of linear $\operatorname{plot}(\eta=1)$ is $-1 / 2$ in agreement with $T_{K}=D e^{-\frac{1}{2 J}}$.

\section{SUMMARY}

In summary, we have studied the Kondo effect in a Luttinger Liquid in presence of a scalar potential. We have obtained the scaling equations up to the second order for an arbitrary Luttinger interaction strength by renormalizing the boson fields.

The ferromagnetic fixed point is studied using an asymptotic Hamiltonian. It is shown that a triplet bound state can be formed in agreement with the recent Bethe ansatz calculation without invoking the nearest neighbor spin interaction. The Luttinger interaction induce the triplet state to break the local SU(2) symmetry about the impurity spin contrary to the result obtained by Furusaki and Nagaosa. The magnetic interaction and the potential scattering do not interplay and the triplet state is sustained for a weak scalar potential, $|J|>4 \omega$. The Kondo temperature for arbitrary strength of the Luttinger interaction is calculated. The result shows a clear crossover behavior from an exponential to a power law type.

Acknowledgement

This work is partially supported by the Korea Research Foundation(99-005-D00011).

[1] Haldane F D M 1981 J. Phys. C:Solid St. Phys. 142585

[2] Emery V J 1979 in Highly Conducting One-dimensional Solids edited by Devreese J T et. al. ( New York:Plenum)

[3] Voit J 1995 Rep. Prog. Phys. 58977

[4] Gogolin A O, Nersesyan A A and Tsvelik A M 1998 Bosonization and Strongly Correlated Systems(Cambridge: Cambridge University)

[5] Schulz H J, Guniberti G and Pieri P cond-mat 9807366

[6] Lee D-H and Toner J 1992 Phys. Rev. Lett. 693378

[7] Furusaki A and Nagaosa N 1994 Phys. Rev. Lett. 72892

[8] Wang Y and Voit J 1996 Phys. Rev. Lett. 774934

[9] Wang Y and Eckern U 1999 Phys. Rev. B 596400

[10] Egger R and Komnik A 1998 Phys. Rev. B 5710620

[11] Fisher M P A and Zwerger W 1985 Phys. Rev. B 326190

[12] Giamarchi T and Schulz H J 1989 Phys. Rev. B 394620

[13] Stone M 1994 Bosonization(World Scientific)

[14] Nomura K 1995 J. Phys. A 285451

[15] Wiegmann P B 1978 J. Phys. C:Solid St. Phys. 111583

[16] Khaliullin G and Fulde P 1995 Phys. Rev. B 529514

[17] Doniach S and Sondheimer E H 1974 Green Functions for Solid State Physicists(Benjamin)

[18] Auerbach A 1994 Interacting Electrons and Quantum Magnetism(Springer) 


\section{Figures}

Fig. 1 The coupling constant $J_{F, B}$ for several Luttinger interaction strength $\eta$. The magnitude of slope and the value of $\left|J_{F}+J_{B}\right|$ become larger as the interaction strength grows larger. The slope of the dotted line is -1 .

Fig. 2 The Kondo temperature as a function of the backward scattering coupling constant, $J_{B}$, for several Luttinger interaction strength $\eta$, where $T_{K}^{o}$ is the Kondo temperature for each $\eta$ at $J_{B} / D=0.1$. The linear to nonlinear crossover is clearly shown. Note the log-log scale of the graph. The inset shows the Kondo temperature as a function of $J_{B}^{-1}$. The linear curve for $\eta=1$ clearly shows the $T_{K}=D e^{-\frac{1}{2 J}}$ behavior. 


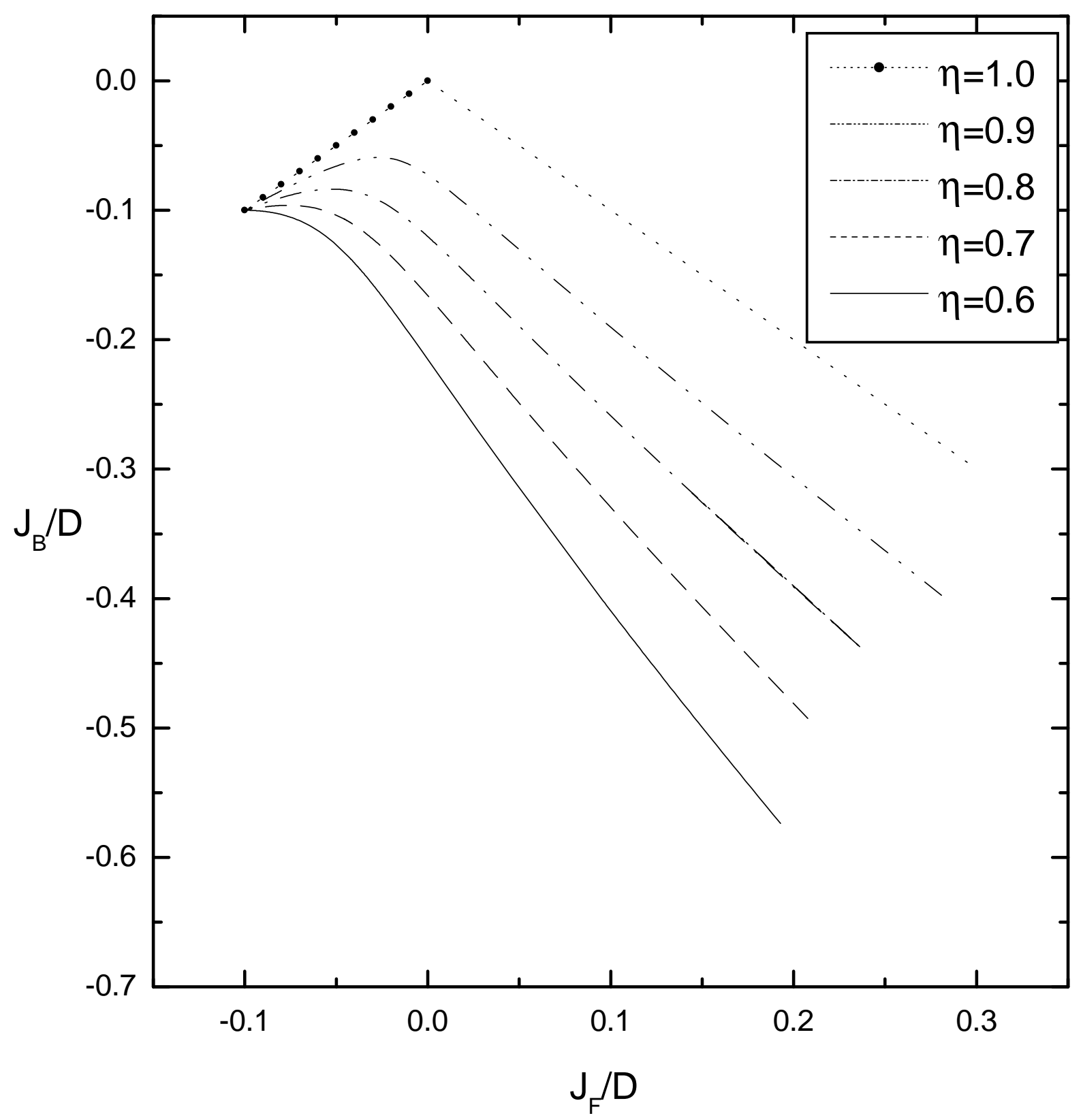

Figure 1 


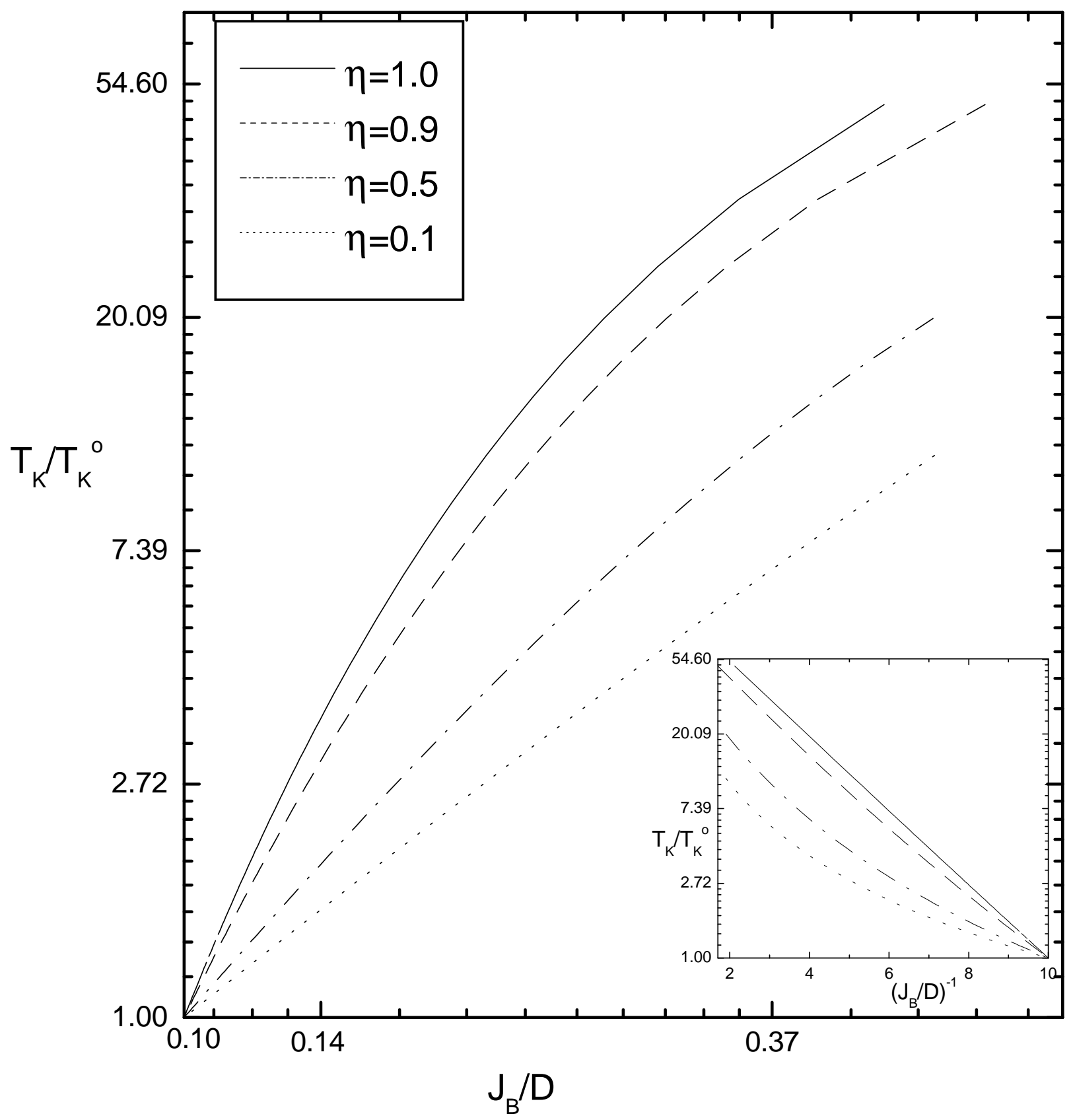

Figure 2 